\title{
INFLUÊNCIA DO CICLO LUNAR NA CAPTURA DE AEDES SCAPULARIS (DIPTERA, CULICIDAE) NA MATA ATLÂNTICA DO PARANÁ ${ }^{1}$
}

\author{
Adson Luís Sant'Ana² \\ Ana Leuch Lozovei ${ }^{3}$
}

\begin{abstract}
INFLUENCE OF THE LUNAR CYCLE ON THE TRAPING OF AEDES SCAPULARIS (DIPTERA, CULICIDAE) IN THE ATLANTIC FOREST OF PARANÁ. From October 1995 to January 1996 Aedes (Ochlerotatus) scapularis (Rondani, 1948) was studied in a small area of the Atlantic Forest, Serra do Marumbi, Morretes, Paraná, Brazil. Adults were captured with Shannon light traps for 48 nights, representing 12 nights for seven time intervals. Traps were operated from dusk to dawn. The influences on flight activity of the lunar phase, ambient temperature, relative humidity, and rainfall were analyzed. Ae. scapularis had a flight behaviour different in each of the four lunar phases. In relation to flight period, the species showed higher activity during the first three lunar intervals. Among the climatic variables, temperature and relative humidity positively influenced flight activity during the night.
\end{abstract}

KEYWORDS. Aedes (Ochlerotatus) scapularis, Culicidae, Diptera, lunar phases, traping.

\section{INTRODUÇÃO}

A atividade de vôo de mosquitos está associada à dispersão, formação de grupo (enxameagem), acasalamento, alimentação, postura ou fuga de circunstâncias desfavoráveis. De acordo com JANOUSEK \& OLSON (1994), numerosos fatores podem influenciar, positiva ou negativamente, a atividade de vôo de mosquitos. $\mathrm{O}$ aumento da umidade resulta em maior atividade de vôo, por exemplo, em Aedes (Aediomorphus) vexans (Meigen, 1830) e em Culex (Culex) nigripalpus Theobald, 1901, conforme Dow $\&$ Gerrish (1970). Outro fator que favorece a atividade de mosquitos é a temperatura (Bradley \& McNeel, 1935; Bidlingmayer, 1974; Carrol \& Bourg, 1977). Ocorre o contrário com a presença de alta velocidade do vento (BradLEY \& McNeel, 1935; BidLINGMAYER, 1974; SERVICE, 1980).

1. Parte da dissertação de mestrado, Curso de Pós-Graduação em Ciências Biológicas, Universidade Federal do Paraná.

2. Departamento de Parasitologia Universidade Federal de Minas Gerais, Av. Pres. Antônio Carlos, 6627, Pampulha, CEP 31270-901, Belo Horizonte, MG. (asantana@mono.icb.ufmg.br). (Bolsista CAPES).

3. Departamento de Patologia Básica, Setor de Ciências Biológicas, Universidade Federal do Paraná, Caixa Postal 19031, CEP 81531-990, Curitiba, PR, Brasil. (alozovei@bio.ufpr.br) 
A intensidade luminosa é um dos fatores importantes na atividade de vôo de culicídeos (Provost, 1959). Chadee (1992), em estudo de Anopheles (Kerteszia) bellator Dyar \& Knab, 1906, Foley et al. (1992), na captura de An. (Cellia) annulipes Walker, 1856 e Pandian \& Chandrashekaran (1980) em pesquisas de Armigeres (Leicesteria) subalbatus Joshi, Pradhan \& Darsie, 1965, observaram maior atividade de vôo das espécies durante as noites de lua cheia. MiLler et al. (1970), capturando diferentes espécies de Anopheles e Culex, Hosfall (1943), trabalhando com espécies de Anopheles, Ribbands (1946), com An. (Cellia) funestus Giles, 1900 e Rubio-Palis (1992), com An. (Nyssorhynchus) nuneztovari Gabaldón, 1940, constataram atividades mais intensas durante a lua nova. NASCI \& EDMAN (1981), em estudos com Culiseta (Allotheobaldia) melanura (Coquillett, 1902) e Singh \& Mishra (1996), em capturas de An. (Cellia) culicifacies Giles, 1901, não encontraram influências significativas das fases lunares. As respostas diferentes a fases lunares, nos diferentes locais de coleta, são devidas à interferência de um conjunto de variáveis ambientais, climáticas e endógenas dos insetos, inclusive das espécies hematófagas veiculadoras de patógenos e não-vetoras, que podem apresentar reações distintas.

Aedes (Ochlerotatus) scapularis (Rondani, 1948) é vetor potencial de vários arbovírus, como encefalite eqüina venezuelana e febre amarela, que acometem humanos e animais (ARnell, 1976; Forattini et al., 1995). A espécie também foi tida vetor secundário de filária bancroftiana (RACHOU et al., 1958) e ainda suspeita de veicular arbovírus da encefalite Rocio no Vale do Ribeira, sudeste do Brasil (FoRATTINI et al., 1981) e, em condições experimentais, teve sua competência vetorial comprovada (Mitchell \& Forattini, 1984; Mitchell et al., 1986). Outros arbovírus veiculados por esta espécie têm sido isolados em condições naturais no norte do Brasil e em Trinidad (ARnell, 1976; Hervé et al., 1986). Recentemente foi investigada como vetor de Dirofilaria immitis (Leidy, 1856) (AHID \& LourençO-DE-OliveIRA, 1999). A espécie em questão é eclética e oportunista quanto ao hospedeiro. Agride o homem com muita voracidade, mas tem grande tendência de investir com maior intensidade ainda contra os mamíferos. É freqüente nas florestas secundárias e nos ambientes alterados, extradomiciliares e mesmo peridomiciliares.

Segundo Forattinı et al. (1995), apresenta forte tendência à endofilia e domiciliação e isto reveste-se de importância epidemiológica.

O objetivo é estudar a atividade de vôo de Ae. scapularis durante as noites das quatro fases lunares e as variáveis climáticas da Serra do Marumbi, interior da Mata Atlântica.

\section{MATERIAL E MÉTODOS}

A área de estudo localiza-se ao sopé da Serra do Marumbi, dentro da Mata Atlântica no Estado do Paraná, sul do Brasil, a $6 \mathrm{~km}$ a noroeste da cidade de Morretes e a $2 \mathrm{~km}$ ao sul do distrito de Porto de Cima, na planície litorânea (25 $30^{\prime}$ S, 48 49' W; 10 m de altitude). As capturas de Ae. scapularis foram efetuadas de outubro de 1995 a janeiro de 1996, em 48 noites, durante quatro meses consecutivos, do início do crepúsculo vespertino até o término do crepúsculo matutino, durante as quatro fases do ciclo lunar. Foram destinadas 12 noites para cada fase da lua.

As capturas foram efetuadas com armadilha de Shannon, com uma fonte luminosa fluorescente de $20 \mathrm{~W}$ ligada a uma bateria de $52 \mathrm{~A}$, que era recarregada antes de cada coleta por um carregador de baterias doméstico. Nas paredes da armadilha, a fim de manter a constância em torno de 70 lux, a luminosidade foi mensurada com luxímetro portátil alimentado por bateria. A armadilha foi instalada uma hora antes do crepúsculo vespertino e 
retirada uma hora depois do matutino, orientados pela tabela de horários do AlmANAQUE NÁUTICO (1995-1996), que traz as fases lunares dos dias do ano e a tabela com valores preestabelecidos que, combinados com a latitude e a longitude de Morretes, possibilitaram os cálculos das horas reais do início e do fim de cada crepúsculo. Houve, portanto, sete intervalos horários com duração de duas horas cada um: intervalo 1 , crepúsculo vespertino e parte da noite, a seguir intervalos 2 a 6 de duração só noturna e o intervalo 7 , crepúsculo matutino e parte do dia. Os intervalos 1,2, 3 e 4 vêm do crepúsculo vespertino até cerca da meia-noite. Os intervalos 5, 6e 7, de meia-noite em diante até o início do dia seguinte. Este procedimento foi necessário para adequar o arranjo dos crepúsculos.

Dentro da armadilha, os mosquitos foram recolhidos durante 15 minutos finais de cada intervalo horário de duas horas através de um sugador manual movido a 8 pilhas alcalinas. Anotavam-se os dados das variáveis climáticas, a temperatura, a umidade relativa do ar e a pluviosidade. As duas primeiras variáveis ambientais eram verificadas com termômetro-higrômetro, enquanto a quantidade de chuva, quando houvesse, com pluviômetro e proveta padrões. No laboratório, os culicídeos foram identificados pelas chaves taxonômicas de Forattini (1965a, b) e SALLUm et al. (1988). A abreviatura dos nomes genéricos seguiu as normas de REINERT (1975). Alguns exemplares da espécie foram depositados na “Coleção Pe. Jesus Santiago Moure", Departamento de Zoologia, Universidade Federal do Paraná, Curitiba ( DZUP).

Para verificar a significância da influência das variáveis mensuradas, nos resultados obtidos foram empregados alguns testes estatísticos não-paramétricos (SIEGEL, 1975), como Friedman (ANOVA) e Kendall, comparações múltiplas em caso de a hipótese Ho ser rejeitada pelo teste ANOVA e ainda o Wilcoxon Matched Pairs Test.

\section{RESULTADOS E DISCUSSÃO}

O maior número de exemplares de Ae. scapularis ocorreu na lua nova $(35,53 \%)$ sendo 52 fêmeas e 2 machos, seguindo-se a lua cheia $(34,21 \%)$ com 47 fêmeas e 5 machos, a lua quarto crescente $(18,42 \%)$ com 26 fêmeas e 2 machos e, por último, a lua quarto minguante $(11,84 \%)$ com 13 fêmeas e 5 machos. Classificando as fases lunares pela maior quantidade de exemplares, em ordem decrescente, apresentam-se a lua nova, a cheia, a quarto crescente e a quarto minguante (tab. I). O teste de Friedman e comparações múltiplas evidenciaram haver diferença entre as capturas da lua quarto minguante com as da lua cheia, a um nível de significância pouco maior que $10 \%$.

Considerando os dados das variáveis climáticas, as médias dos intervalos horários da temperatura, da umidade relativa do ar e da pluviosidade havidas durante as noites de coleta, ocorria queda da temperatura à medida do avançar da noite, a partir do intervalo 1 até o 6, em todas as fases lunares, com exceção das últimas horas, no sétimo intervalo, que sempre acusava elevação. Em geral, o gradiente de temperatura oscilou de $22,2^{\circ} \mathrm{C}$ a $15,9^{\circ} \mathrm{C}$, com uma diferença, em média, de $6,3^{\circ} \mathrm{C}$ entre a máxima e a mínima registradas. Nas quatro fases lunares, a temperatura aumentava sensivelmente do penúltimo horário em relação ao último. Nestes dois horários, em ordem decrescente, as diferenças foram de $5,2^{\circ} \mathrm{C}$ na quarto minguante, $2,2^{\circ} \mathrm{C}$ na nova, $1,9^{\circ} \mathrm{C}$ na cheia e $1,7^{\circ} \mathrm{C}$ na quarto crescente. Estatisticamente, foi significativa a influência da temperatura sobre a atividade de vôo de Ae. scapularis. A umidade relativa do ar manteve-se bem elevada durante as noites de pesquisa, oscilando, em média, de $97,1 \%$ a $89,4 \%$, com diferença de 7,7\% entre máximo e mínimo (tab. I). A atividade de vôo de $A e$. scapularis estatisticamente sofreu significativa influência da umidade relativa do ar. Com relação às precipitações pluviométricas, observou-se escassez ou mesmo ausência total de chuva nas noites de pesquisa. Os índices pluviométricos variaram, em média, de 0,25 mm a zero (tab. I). Não houve condições, portanto, de determinar influência ou não desta variável na atividade de vôo da espécie em questão.

Williams (1935), pesquisando sobre a luminosidade lunar, encontrou entre os 
Tabela I. Total capturado de Ae. scapularis em 48 noites nas quatro fases lunares e médias de temperatura ambiente, umidade relativa do ar e pluviosidade nos intervalos horários, Mata Atlântica, Serra do Marumbi, Morretes, Paraná, Brasil, outubro de 1995 a janeiro de 1996.

\begin{tabular}{|c|c|c|c|c|c|c|}
\hline $\begin{array}{l}\text { Fases de lua } \\
\text { e intervalos } \\
\text { horários de } \\
\text { coletas }\end{array}$ & \multicolumn{3}{|c|}{ Aedes scapularis } & \multirow{2}{*}{$\begin{array}{l}\text { temperat. } \\
\text { ambiente } \\
\text { média } \\
\left({ }^{\circ} \mathrm{C}\right)\end{array}$} & \multirow{2}{*}{$\begin{array}{l}\text { umidade } \\
\text { relativa } \\
\text { média } \\
(\%)\end{array}$} & \multirow{2}{*}{$\begin{array}{l}\text { pluviosi- } \\
\text { dade } \\
\text { média } \\
\left(\mathrm{mm}^{3}\right)\end{array}$} \\
\hline \multicolumn{4}{|l|}{ cheia } & & & \\
\hline 1 & 10 & 1 & 11 & 22,2 & 90,7 & 0,08 \\
\hline 2 & 13 & 3 & 16 & 21,4 & 93,5 & 0,08 \\
\hline 3 & 9 & 1 & 10 & 20,5 & 95,2 & 0,16 \\
\hline 4 & 5 & 0 & 5 & 19,9 & 96,2 & 0,25 \\
\hline 5 & 7 & 0 & 7 & 19,6 & 96,7 & 0,16 \\
\hline 6 & 3 & 0 & 3 & 19,2 & 95,4 & 0,16 \\
\hline 7 & 0 & 0 & 0 & 21,1 & 93,6 & 0,16 \\
\hline subtotal & 47 & 5 & 52 & 20,5 & 94,5 & 0,15 \\
\hline \multicolumn{7}{|l|}{ minguante } \\
\hline 1 & 6 & 2 & 8 & 21,3 & 90,7 & 0,16 \\
\hline 2 & 4 & 2 & 6 & 20,5 & 93,5 & 0 \\
\hline 3 & 1 & 0 & 1 & 19,7 & 95,2 & 0 \\
\hline 4 & 0 & 1 & 1 & 19,1 & 96,2 & 0,08 \\
\hline 5 & 0 & 0 & 0 & 18,0 & 97,0 & 0,08 \\
\hline 6 & 2 & 0 & 2 & 15,9 & 95,4 & 0,08 \\
\hline 7 & 0 & 0 & 0 & 21,0 & 93,6 & 0 \\
\hline subtotal & 13 & 5 & 18 & 19,3 & 94,5 & 0,07 \\
\hline \multicolumn{7}{|l|}{ nova } \\
\hline 1 & 19 & 0 & 19 & 21,1 & 89,4 & 0 \\
\hline 2 & 11 & 1 & 12 & 20,2 & 91,0 & 0 \\
\hline 3 & 11 & 1 & 12 & 19,5 & 93,2 & 0 \\
\hline 4 & 4 & 0 & 4 & 18,9 & 94,3 & 0 \\
\hline 5 & 4 & 0 & 4 & 18,5 & 94,8 & 0 \\
\hline 6 & 2 & 0 & 2 & 18,2 & 95,1 & 0 \\
\hline 7 & 1 & 0 & 1 & 20,4 & 93,7 & 0 \\
\hline subtotal & 52 & 2 & 54 & 19,5 & 93,1 & 0 \\
\hline \multicolumn{7}{|l|}{ crescente } \\
\hline 1 & 7 & 1 & 8 & 20,2 & 97,0 & 0,25 \\
\hline 2 & 5 & 0 & 5 & 19,7 & 95,5 & 0,25 \\
\hline 3 & 4 & 0 & 4 & 19,2 & 96,1 & 0,08 \\
\hline 4 & 5 & 1 & 6 & 19,1 & 96,7 & 0,08 \\
\hline 5 & 2 & 0 & 2 & 18,9 & 97,0 & 0,25 \\
\hline 6 & 2 & 0 & 2 & 18,7 & 97,1 & 0 \\
\hline 7 & 1 & 0 & 1 & 20,4 & 96,1 & 0,08 \\
\hline subtotal & 26 & 2 & 28 & 19,4 & 96,5 & 0,14 \\
\hline Total & 138 & 14 & 152 & & & \\
\hline$\%$ & 90,79 & 9,21 & 100 & & & \\
\hline
\end{tabular}

noctuídeos (Lepidoptera) capturados na lua nova e os capturados na lua cheia a proporção de 3:1 em noites de céu aberto e de 2:1 em noites nubladas. BIDLINGMAYER (1964) salientou que os efeitos diretos da luminosidade lunar sobre a atividade de vôo de insetos não têm sido extensivamente estudados, enquanto são mais conhecidos os efeitos indiretos sobre a captura de mosquitos, utilizando armadilha luminosa. Vários trabalhos têm revelado que as capturas são mais reduzidas durante o período de lua cheia em contraste com o da lua nova (Bradley \& McNeel, 1935; Hosfall, 1943; Pratt, 
1948; Provost, 1959; Bowden, 1973; Rubio-Palis, 1992). Nesta pesquisa praticamente não houve diferença significativa no número de mosquitos entre as mencionadas duas fases lunares. Isto se deve à armadilha luminosa sofrer diminuição de sua eficiência física durante a fase de lua cheia, competindo com a luminosidade lunar, e exercendo menos atratividade. Alguns trabalhos revelam haver muitas espécies de mosquitos mais ativas nas noites de lua cheia, se comparadas com períodos de menor luminosidade lunar. Bidlingmayer (1964), Davies (1975), Charlwood et al. (1986) e Chadee (1992) sugerem que na lua cheia exista prolongamento da intensidade luminosa, fato que, segundo AlLen et al. (1987), favoreceria a habilidade de culicídeos em localizar os hospedeiros e os locais de oviposição.

O comportamento de Ae. scapularis apresentou-se diferente nas fases da lua e também nos diferentes intervalos horários (tab. I, fig. 5). Com relação às fases, percebese nitidamente que, na lua cheia (fig. 1), a espécie foi coletada em todos os intervalos horários, menos no último. No intervalo 2, apresentou maior número de indivíduos (tab. I). Pelo teste Friedman, a hipótese Ho foi rejeitada, revelando que pelo menos um intervalo horário difere dos demais $(\mathrm{P}<0,05)$. Apesar de existir diferença, não foi possível detectá-la pelas comparações múltiplas. Pelo Wilcoxon Matched Pairs Test, a hipótese Ho foi rejeitada, ou seja, a análise estatística permitiu verificar que a quantidade de capturados nos três primeiros intervalos difere dos quatro últimos em nível de significância de $10 \%$.

Na lua quarto minguante, Ae. scapularis foi coletada nos intervalos de 1 a 4 e 6 , não o sendo nos 5 e 7.

O maior número de exemplares ocorreu no intervalo 1 , seguido do intervalo 2 (fig. 2). O teste de ANOVA rejeitou a hipótese Ho ( $\mathrm{P}<0,002)$. Neste caso, apesar de existirem diferenças significativas, não foi possível detectar pelas comparações múltiplas. Pelo Wilcoxon Matched Pairs Test, a hipótese Ho foi rejeitada ao nível de significância de $5 \%$. Observa-se que a espécie-alvo nas capturas de lua nova ocorreu em todos os intervalos horários, sobressaindo-se no primeiro (fig. 3). O teste ANOVA rejeitou a hipótese Ho $(\mathrm{P}<0,07)$. Apesar de existirem diferenças estatisticamente significativas, não foi possível detectá-las pelas comparações múltiplas. O Wilcoxon Matched Pairs Test também rejeitou a hipótese Ho ao nível de 7\%. Na lua quarto crescente, Ae. scapularis ocorreu em todos os intervalos horários, apresentando maior número de exemplares nos intervalos 1 e 4 (fig. 4). Estatisticamente, não houve diferenças significativas entre os intervalos horários nesta fase lunar. Computando os exemplares de Ae. scapularis nas quatro fases lunares e distribuindo-os nos sete intervalos horários, observa-se o decréscimo paulatino na quantidade de indivíduos na medida do avançar noite adentro até ao amanhecer (fig. 5).

Algumas espécies de mosquitos podem apresentar mais de um pico em capturas de 24 horas consecutivas. Esta variação pode ser bimodal crepuscular, alternância de ocorrência no amanhecer e no anoitecer ou durante o mesmo período do dia ou da noite. Ae. scapularis, nos estudos de GuIMARÃES \& Victorio (1986), apresentou o segundo tipo de distribuição, embora também tenha sido capturada em elevados números em diferentes horas do dia. De modo geral, esta espécie apresenta aumento de sua atividade hematofágica no início do crepúsculo vespertino. Observação semelhante também foi verificada por RACHOU et al. (1958). ForATTINI et al. (1981) ressaltaram que apesar de ter hábitos diurnos, no Vale do Ribeira, São Paulo, Ae. scapularis demonstrou intensa 


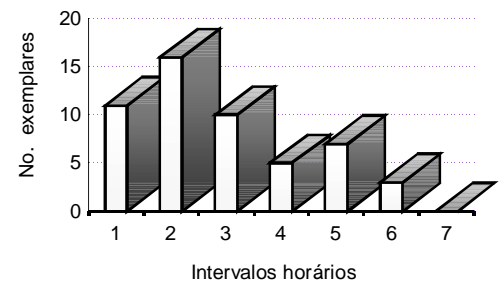

1

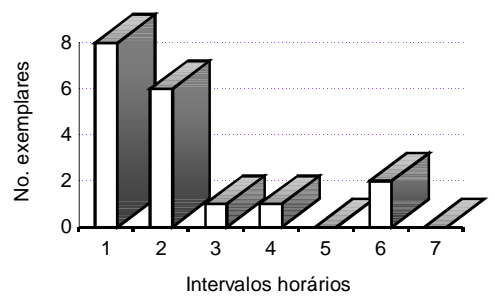

2
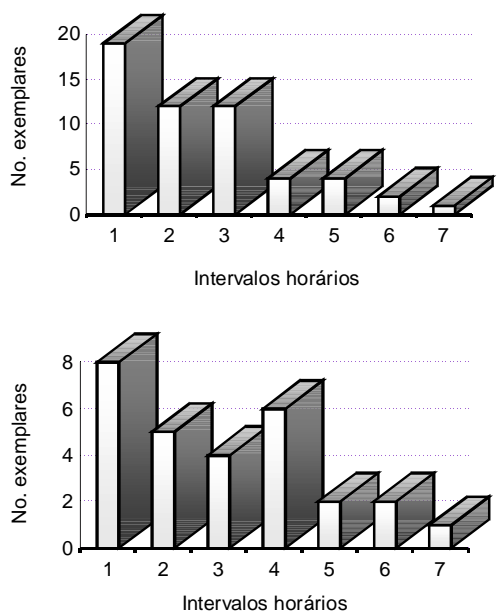

4

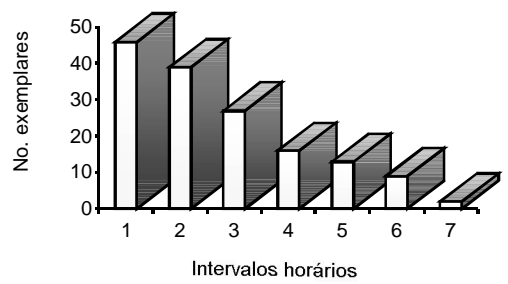

5

Figuras 1-5. Total de indivíduos capturados de Aedes scapularis em sete intervalos horários, Mata Attântica, Morretes, Paraná, Brasil, outubro 1995 a janeiro 1996, nas luas: 1, cheia; 2, minguante; 3 , nova; 4, crescente; 5 , nas quatro fases lunares, em 48 noites. atividade noturna com pico máximo endocrepuscular vespertino. De acordo com Degallier et al. (1978), a sua presença restringiu-se quase exclusivamente às capturas diurnas e LOURENÇO-DE-OLIVEIRA \& Silva (1985) consideram eclética a espécie com aumento da densidade no final do crepúsculo vespertino, igual ao observado na presente investigação.

$\mathrm{Na}$ área do presente estudo, Ae. scapularis apresentou atividade noturna de vôo mais eficiente, em ordem decrescente, nas luas nova, cheia, quarto crescente e, por último, na quarto minguante (tab. I).

Depreende-se que os diferentes desempenhos de vôo durante as quatro fases lunares são fruto de critérios fisiológicos e bionômicos específicos, aqui registradas pela primeira vez. Ainda, nas fases lunares de cheia, nova e quarto minguante, prevalece uma maior atividade de vôo nos três primeiros intervalos horários, com melhor desempenho em capturas no crepúsculo vespertino, atividade influenciada positivamente pelas temperatura e umidade relativa do ar em níveis estatisticamente significativos. 
Agradecimentos. Aos professores Ricardo Sandes Ehlers e Joel Maurício Correia da Rosa, Departamento de Estatística, Universidade Federal do Paraná, pelo auxílio na elaboração conjunta dos testes estatísticos.

\section{REFERÊNCIAS BIBLIOGRÁFICAS}

Ahid, S. M. M. \& Lourenço-DE-Oliveira, R. 1999. Mosquitos vetores potenciais de Dirofilariose canina na Região Nordeste do Brasil. Revta Saúde públ., São Paulo, 33 (6): 560-565.

Allen, S. A.; Day, J. F. et al. 1987. Visual ecology of biting flies. A. Rev. Ent., Palo Alto, 32: $297-316$.

Almanaque Náutico, 1995-1996. Rio de Janeiro, Ministério da Marinha, Diretoria de Hidrografia e Navegação. $287 \mathrm{p}$.

Arnell, J. H. 1976. Mosquito Studies (Diptera: Culicidae). XXXIII. A revision of the Scapularis Group of Aedes (Ochlerotatus). Contr. Am. Ent. Inst., Washington, 13 (3): 1-44.

BiDLINGMAYER, W. L. 1964. The effect of moonlight on the flight activity of mosquitoes. Ecology, Brooklyn, 45: 87-94.

. 1974. The influence of environmental factors and physiological stage on flight patterns of mosquitoes taken in the vehicle aspirator and truck suction bait and new jersey light traps. J. med. Ent.., Lenham, 11: 19-146.

Bowden, J. 1973. The significance of moonlight in photoperiodic responses of insects. Bull. Ent. Loyola Coll., Madras, 62: 605-612.

Bradley, G. H. \& McNeel, T. E. 1935. Mosquito collections in Florida with the new jersey light trap. J. econ. Ent., Geneva, 28: 780-786.

Carrol, M. K. \& Bourg, M. 1977. The night-time flight and relative abundance of fiften species of louisiana mosquitoes. Mosq. News, New Brunswick, 37: 661-664.

CHADEE, D. D. 1992. Indoor and outdoor host-seeking rhythms of Anopheles bellator (Diptera: Culicidae) in Trinidad, West Indies. J. med. Ent., Lenham, 29 (3): 567-569.

Charlwood, J. D.; PARu, R. et al. 1986. Influence of moonlight and gonotrophic age on biting activity of Anopheles farauti (Diptera: Culicidae) from Papua New Guinea. J. med. Ent., Lenham, 23: 132-135.

DAviEs, J. B. 1975. Moonlight and the biting activity of Culex (Melanoconion) portesi Sevenet \& Abonnenc and Culex (Melanoconion) taeniopus Dyar \& Knab (Diptera: Culicidae) in Trinidad forests. Bull. ent. Res., London, 65:81-96.

Degallier, N.; Pajot, F. X. et al. 1978. Rythmes d'activité des culicides de la Guyane Française (Diptera: Culicidae). Cah. ORSTOM., Sér. Ent. Med. Parasitol., Bondy, 16: 73-84.

Dow, R. P. \& Gerrish, G. M. 1970. Day-to-day change in relative humidity and activity of Culex nigripalpus (Diptera: Culicidae). Ann. ent. Soc. Am., Columbus, 63: 995-999.

Foley, D. H.; Barnes, A. et al. 1992. Anopheles annulipes Walker (Diptera, Culicidae) at griffith, New-SouthWales. 4. Phenology of 2 sibling species. J. aust. Ent. Soc., Sydney, 31: 91-96.

Forattini, O. P. 1965a. Entomologia Médica. São Paulo, Universidade de São Paulo. v. 2 , 506 p. 1965b. Entomologia Médica. São Paulo, Universidade de São Paulo. v. 3, 416 p.

Forattini, O. P.; Gomes, A. C. et al. 1981. Observações sobre atividade de mosquitos Culicidae, em mata residual no Vale do Ribeira, São Paulo, Brasil. Revta Saúde públ., São Paulo, 15: 557-86.

Forattini, O. P.; Kakitani, I. et al. 1995. Studies on mosquitoes (Diptera: Culicidae) and anthropic environment 9-Synanthropy and epidemiological vector role of Aedes scapularis in South-Eastern Brazil. Revta Saúde públ., São Paulo, 29 (3): 199-207.

Guimarães, A. E. \& Victorio, V. M. N. 1986. Mosquitos no Parque Nacional da Serra dos Órgãos, Estado do Rio de Janeiro, Brasil. 3. Preferência horária para hematofagia. Mems Inst. Oswaldo Cruz, Rio de Janeiro, 81 (1): 93-103

Hervé, J. P.; Degallier, N. et al. 1986. Arboviroses - Aspectos ecológicos. In: Instituto Evandro Chagas. 50 anos de contribuição às Ciências Biológicas e à Medicina Tropical. Belém, Fund. Serv. Saúde Pública. v. 1, $529 \mathrm{p}$.

Hosfall, W. R. 1943. Some responses of the malaria mosquito to light. Ann. ent. Soc. Am., Columbus, 36 (1): 41-45.

JANOUSEK, T. E. \& Olson, J. K. 1994. Effect of a lunar eclipse on the activity of mosquitoes in the upper gulf coast of texas. J. Am. Mosq. Contr. Ass., Washington, 10 (2): 222-224.

Lourenço-De-Oliveira, R. \& Silva, T. F. 1985. Alguns aspectos da ecologia dos mosquitos (Diptera: Culicidae) de uma área de planície (Granjas Calábria), em Jacarepaguá, Rio de Janeiro. 3. Preferência horária das fêmeas para o hematofagismo. Mems Inst. Oswaldo Cruz, Rio de Janeiro, 80 (2): 195 -201.

Miller, T. A.; Stryker, R. G. et al. 1970. The influence of moonlight and other environmental factors on the 
abundance of certain mosquito species in light-trap collections in Thailand. J. med. Ent., Lenham, 7 (5): 555561.

Mitchell, C. J.\& Forattini, O. P. 1984. Experimental transmission of Rocio encephalites virus by Aedes scapularis (Diptera: Culicidae) from the endemic zone in Brazil. J. med. Ent. Lenham, 21: 34-37.

Mitchell, C. J.; Forattini, O. P. et al. 1986. Vector competence experiments with Rocio virus and three mosquito species from the epidemic zone in Brazil. Revta Saúde públ., São Paulo, 20: 171-177.

Nasci, R. S. \& Edman, J. D. 1981. Vertical and temporal flight activity of the mosquito Culiseta melanura (Diptera, Culicidae) in southeastern Massachusetts. J. med. Ent., Lenham, 18 (6): 501-504.

Pandian, R. S. \& ChandrasheKaran, M. K. 1980. Rhythms in the biting behaviour of a mosquito Armigeres subalbatus. Oecologia, Berlim, 47 (1): 89-95.

Pratt, H. J. 1948. Influence of the moon on light trap collections of Anopheles albimanus in Puerto Rico, J. natn. Malar. Soc., Columbia, 7: 212-220.

Provost, M. W. 1959. The influence of moonlight on light-trap catches of mosquitoes. Ann. ent. Soc. Am., Washington, 52: 261-271.

Rachou, R. G.; Moura Lima, M. et al. 1958. Alguns dados sobre o comportamento de mosquitos de Ponta Grossa (Florianópolis, Santa Catarina). Revta bras. Malar. Doenç. trop., Rio de Janeiro, 10: 417-427.

ReINERT, J. F. 1975. Mosquito generic and subgeneric abbreviations (Diptera: Culicidae). Mosq. Systematics, 7 (2): $105-110$.

RibBands, C. R. 1946. Moonlight and house hauting habits of female Anopheles in W. Africa. Bull. ent. Res., London, 36: 395-415.

RuBio-PaLIS, Y. 1992. Influence of moonlight on light trap catches of the malaria vector Anopheles nuneztovari in Venezuela. J. Am. Mosq. Contr. Ass., Washington, 8 (2): 178-180.

Sallum, M. A. M.; Uramoto, K. et al. 1988. Redescription and resurrection from synonymy of Aedes (Ochlerotatus) rhyacophilus Costa Lima, 1933. Mems Inst. Oswaldo Cruz., Rio de Janeiro, 83 (1): 6777.

SERVICE, M. W. 1980. Effects of wind on the behaviour and distribution of mosquitoes and blackflies. Int. J. Biomet., Leiden, 24: 347-353.

SIEGEL, S. 1975. Estatística não Paramétrica (para ciências do comportamento). Rio de Janeiro, McGrawHill. 333 p.

SingH, N. \& Mishra, A. K. 1996. Influence of moonlight on light-trap catches of the malaria vector Anopheles culicifacies (Diptera, Culicidae) in central India. Bull. ent. Res., London, 86: 475-479.

WilLiams, C. B. 1935. The times of activity of certain nocturnal insects, chiefly Lepidoptera, as indicated by light trap. Trans. R. ent. Soc., London, 83 (4): 523-525.

Recebido em 20.03.2000; aceito em 04.12.2000. 